\title{
Prevalence of gestational diabetes mellitus and its maternal and fetal outcomes
}

Sir,

We hereby report the results of a prospective cohort study where we compared the association of various demographic factors and gestational diabetes mellitus (GDM), determined the prevalence of GDM and its maternal and fetal outcomes.

This study includes all patients who fulfilled the inclusion criteria: antenatal women between 24-28 weeks of gestation both inpatients and outpatients and willing for safe confinement in the study setting after obtaining informed written consent. Exclusion criteria consisted of pregestational diabetes, pregnancies with multifetal gestation, pregnancy with hypertension, autoimmune conditions such as systemic lupus erythematosus, chronic conditions such as congestive heart failure, chronic renal failure and active tuberculosis and antenatal women on steroid therapy for any condition.

A total of 254 women fulfilling the selection criteria were randomly selected. After obtaining written consent, a detailed history followed by complete general and physical examination were carried out. After explaining the procedure, the participants were asked to consume $75 \mathrm{gm}$ of anhydrous glucose, dissolved in $300 \mathrm{ml}$ of water, irrespective of their last meal. The time of glucose consumption was noted.

After 2 hours, blood samples were drawn from the antecubital vein (within 1-2 min of each other). Blood samples were collected in sodium fluoride/disodium ethylenediaminetetraacetate (Na2 EDTA) vacutainer tubes to prevent glycolysis. Samples were transported to the laboratory within 1-2 hours and venous plasma glucose was measured in our laboratory using an autoanalyzer.

Plasma glucose was measured by glucose oxidaseperoxidase (GOD-POD) method. If the plasma glucose levels were $\geq 140 \mathrm{mg} / \mathrm{dl}$, the participants were labelled as gestational diabetes mellitus (GDM). Those with plasma glucose $<140 \mathrm{mg} / \mathrm{dl}$ were taken as controls.

Diabetes in pregnancy study group of India (DIPSI) test is done for all sample population in 1st trimester if negative it was repeated at 24-28 weeks. Those who are seen late in 2nd trimester had the test only once. Those diagnosed as GDM were followed by meal plan for two weeks and 2 hour postprandial glucose (PPPG) level checked. If 2 hour PPPG $<120 \mathrm{mg} / \mathrm{dl}$ meal plan continued and if 2 hour PPPG $\geq 120 \mathrm{mg} / \mathrm{dl}$ metformin or insulin started. All the participants were followed up till delivery and the following outcomes were recorded and compared between GDM and non GDM groups.

The parameters studied are age group, parity, family history, past history, prevalence of GDM, gestational age at the time of delivery, maternal outcomes in the form of mode of delivery (spontaneous vaginal/instrumental/caesarean section), fetal outcomes included birth weight, meconium stained liquor, respiratory distress syndrome and congenital anomalies.

The mean age of the study participants was $24.5 \pm 3.6$ years. Majority of the participants belonged to the age group of $20-25$ years $(66.2 \%)$ followed by $26-30$ years $(28.3 \%)$. It was observed that among the age group of 31-35 years, $25 \%$ had GDM while in the age group of 26-30 years, $15.3 \%$ had GDM. Majority of the women, $51.9 \%$ were multigravida while $48.1 \%$ of the participants were primigravida. Among the primigravida participants, $15.6 \%$ had GDM while among the multigravida, $12.9 \%$ had GDM. The observed difference was not statistically significant. Family history of GDM was $9.5 \%$. Among the multigravida women $3 \%$ had previous history of GDM. The prevalence of GDM was $14.2 \%$. Among GDM participants $77.8 \%$ were controlled on medical nutrition therapy, while $13.9 \%$ were controlled on oral hypoglycaemic agents and remaining $8.3 \%$ with insulin therapy. In the GDM group, $63.9 \%$ delivered during the gestational age of $37-39^{+6}$ weeks and $11.1 \%$ delivered during $34-36^{+6}$ weeks of gestation. No GDM cases were delivered before 34 weeks of gestation. Among the GDM participants, $63.9 \%$ underwent caesarean section and $30.6 \%$ underwent spontaneous vaginal delivery and 5.5\% underwent instrumental deliveries. Among the GDM mothers $23.08 \%$ had induced labour and $76.92 \%$ had spontaneous labour. In this study, 63.9\% GDM participants delivered by caesarean section compared to $58.3 \%$ of the non GDM participants. The observed difference was statistically not significant. Among GDM mothers who underwent lower segment caesarean section (LSCS), the most common indication was cephalopelvic disproportion (52.2\%) followed by fetal distress (30.4\%). Among the non GDM mothers, fetal distress was the most common indication $(48.03 \%)$. The mean birth weight of the neonates was $2.8 \pm 0.4 \mathrm{~kg}$. Majority of the participants with GDM were delivered babies between $2.5-3.5 \mathrm{~kg}$ (66.7\%). Among GDM mothers 25\% delivered babies weighing $>3.5 \mathrm{~kg}$ compared to $3.2 \%$ of the non GDM 
mothers. The observed difference was statistically significant. In this study, it was observed that meconium stained liquor was present in $13.9 \%$ of the GDM participants compared to $3.2 \%$ of the non GDM participants. The observed difference was statistically significant. In this study, neonatal intensive care unit (NICU) admissions for RDS were present in $30.6 \%$ of the GDM participants, compared to $11.9 \%$ of the non GDM participants. The observed difference was statistically significant. In this study, 5.5\% neonates were diagnosed with neonatal hypoglycaemia in GDM group compared to $1.8 \%$ in non GDM group. No significant association was observed between hypoglycaemia and GDM. No congenital anomalies were detected in GDM. Our studies like other studies have shown adverse effect on maternal and fetal outcomes. ${ }^{1-4}$

\section{Gude P. Narayanrao ${ }^{1 *}$, R. Kala ${ }^{1}$, A. Kumar $^{2}$}

${ }^{1}$ Department of Obstetrics and Gynaecology, ${ }^{2}$ Department of Pediatrics, District Government Headquarters Hospital, Cuddalore, Tamilnadu, India

*Correspondence to

Dr. Gude P. Narayanrao, E-mail: drprajaktag@gmail.com

\section{REFERENCES}

1. Rajput R, Yadav Y, Nanda S, Rajput M. Prevalence of gestational diabetes mellitus \& associated risk factors at a tertiary care hospital in Haryana. Indian J Med Res. 2013;137:728-33.

2. Mahalakshmi MM, Bhavadharini B, Kumar M, Anjana RM, Shah SS, Bridgette A, et al. Clinical profile, outcomes, and progression to type 2 diabetes among Indian women with gestational diabetes mellitus seen at a diabetes center in south India. Indian J Endocrinol Metab. 2014;18(3):400-6.

3. Prakash GT, Das AK, Habeebullah S, Bhat V, Shamanna SB. Maternal and neonatal outcome in mothers with gestational diabetes mellitus. Indian J Endocr Metab. 2017;21:854-8.

4. Kumari R, Dalal V, Kachhawa G, Sahoo I, Khadgawat R, Mahey R, et al. Maternal and perinatal outcome in gestational diabetes mellitus in a Tertiary Care Hospital in Delhi. Indian $\mathbf{J}$ Endocr Metab. 2018;22:116-20.

Cite this article as: Narayanrao GP, Kala R, Kumar A. Prevalence of gestational diabetes mellitus and its maternal and fetal outcomes. Int J Reprod Contracept Obstet Gynecol 2020;9:5206-7. 